\title{
Meson Loop Corrections to the NJL Model
}

\author{
Francisco Peña, M. Carolina Nemes, \\ Departamento de Fúsica, Instituto de Ciências Exatas, \\ Universidade Federal de Minas Gerais, \\ Belo Horizonte, CEP 30.161-970, C.P. 702, MG, Brazil \\ Alex H. Blin, and Brigitte Hiller \\ Departamento de Física, Universidade de Coimbra, \\ P-3004-516 Coimbra, Portugal \\ Received 26 January, 1999
}

\begin{abstract}
We present a symmetry preserving approach to $1 / N$ ( $N$ being the number of colors) corrections in the Nambu-Jona-Lasinio model. This is achieved through an explicit $1 / N$ expansion where $N$ is considered as a free parameter. Verification and use of the pertinent Ward identity to the next to leading order in $1 / N$ is made in obtaining the Goldberger-Treiman relation. Although the present results are not new, the approach may be useful for deriving higher (than one loop) corrections to the NJL model in a systematic way.
\end{abstract}

\section{Introduction}

The Nambu-Jona-Lasinio (NJL) model [1] has proven to be a valuable tool for our understanding of pion chiral dynamics and several important aspects of chiral symmetry in the domain of low energy hadron physics. Also of theoretical relevance is the fact that nonperturbative methods devised to treat the NJL model turned out to be adequate to study spontaneous symmetry breaking in confining quark models [2] and QCD [3].

This suggests that the study of chirally invariant nonperturbative approximations has considerable interest in its own right. In the context of the Nambu-JonaLasinio (NJL) [1] model the best known nonperturbative method is the one quark loop approximation. It has been applied to study numerous features of strong interaction physics at low energies (for recent reviews see [5]) with striking success. Much less work, however, has been comparatively devoted to implementations of higher order approximations. Among these we can quote [6-10]. These approaches are based on a $1 / N$ expansion, where $N$ denotes the number of colors. As discussed in [10], symmetry properties of the system can be easily violated by an inappropriate choice of di- agrams. Methods which preserve the symmetry properties at higher orders deserve our special attention, refs. $[9,10]$.

The purpose of this paper is to present a mathematical implementation of a $1 / N$ expansion where $N$ is considered as a free parameter. We make use of the Functional Integral Approach and show that a completely systematic expansion can be constructed in such a way that it automatically yields all necessary Feynman graphs to a given order in $1 / N$, being therefore self consistent. Our results coincide with those of refs. $[9,10]$, which are symmetry preserving, to their calculation order. Higher order contributions can be computed in the present scheme. Moreover, we implement the calculation of the pion decay constant within the very same approximation scheme and corrections to it are given by the same means and are on the same footing as corrections to the meson and fermion propagators.

\section{The Model}

Let us start by considering the two flavor N.J model Lagrangian: 


$$
\mathcal{L}=\bar{\Psi}(i \not \partial-\hat{m}) \Psi+\frac{G}{2}\left[(\bar{\Psi} \Psi)^{2}+\left(\bar{\Psi} i \gamma_{5} \tau_{a} \Psi\right)^{2}\right] ; a=1,2,3
$$

where $\tau_{a}$ are the isospin matrices and $\hat{m}$ denotes the current quark mass, which we take to be equal in the $u$ and $d$ quark sectors. It is convenient to redefine the strong coupling constant as $g=G N$ in order to make the number of colors $N$ explicit. It is helpful to repeat some well known techniques in the light of an expansion in terms of $1 / N$ counting: We proceed to bosonize partially the Lagrangian, introducing auxiliary fields $\varphi_{i}$ with the quantum numbers of a scalar $\hat{S}$ and of pseudoscalar $\hat{P}_{a}$ fields, in such a way that the new Lagrangian

$$
\mathcal{L} \rightarrow \mathcal{L}-\frac{1}{2 g}\left[\hat{S}-\frac{g}{\sqrt{N}} \bar{\Psi} \Psi\right]^{2}-\frac{1}{2 g}\left[\hat{P}_{a}-\frac{g}{\sqrt{N}} \bar{\Psi} i \gamma_{5} \tau_{a} \Psi\right]^{2}
$$

has exactly the same dynamical content as the original one. One verifies from the Euler-Lagrange equations of motion

$$
\frac{\delta \mathcal{L}}{\delta \varphi_{i}}-\frac{\delta \mathcal{L}}{\delta\left(\partial_{\mu} \varphi_{i}\right)}=0
$$

that

$$
\hat{S}=\frac{g}{\sqrt{N}} \bar{\Psi} \Psi
$$

and

$$
\hat{P}_{a}=\frac{g}{\sqrt{N}} \bar{\Psi} \tau_{a} i \gamma_{5} \Psi
$$

and therefore all steps are exact. In terms of the new fields the Lagrangian reads

$$
\mathcal{L}=-\frac{1}{2 g} \hat{S}^{2}-\frac{1}{2 g} \hat{P}_{a}^{2}+\bar{\Psi}\left[i \not \partial-\hat{m}+\frac{1}{N}\left(\hat{S}+\hat{P}_{a} \tau_{a} i \gamma_{5}\right)\right] \Psi .
$$

The expectation value of the scalar field in vacuum $\langle\hat{S}\rangle=\sqrt{N} s$ is finite. By shifting the scalar field to a new field $\sigma$ with vanishing vacuum expectation value, $\langle\sigma\rangle=0$ one generates the constituent quark mass $m$, $-m+\sigma / \sqrt{N}=-\hat{m}+\hat{S} / \sqrt{N}$. In terms of the new scalar field $\sigma$ and the pseudoscalar fields $\pi_{a}$ one has

$$
\mathcal{L}=N\left(\frac{-s^{2}}{2 g}\right)+\sqrt{N}\left(\frac{s \sigma}{g}\right)^{2}-\frac{\sigma^{2}}{2 g}-\frac{\pi_{a}^{2}}{2 g}+\bar{\Psi}\left[i \not \partial-m+\frac{1}{\sqrt{N}}\left(\sigma+i \pi \gamma_{5}\right)\right] \Psi ; \pi=\pi_{a} \tau^{a} .
$$


Using functional integral methods, one introduces the generating function for the Green's functions

$$
\mathcal{Z}\left[\eta, \bar{\eta}, j_{\sigma}, j_{\pi_{a}}\right]=\mathcal{N}^{\prime} \int \mathcal{D} \bar{\Psi} \mathcal{D} \Psi \mathcal{D} \sigma \Pi_{a} \mathcal{D} \pi_{a} e^{i\left(S+\bar{\eta} \Psi+\bar{\Psi} \eta+j_{\sigma} \sigma+j_{\pi_{a}} \pi^{a}\right)}
$$

where $S$ denotes the action and $\eta$ and $\bar{\eta}$ are the source terms of the fermionic fields, and $j_{\sigma}, j_{\pi_{a}}$ are the source terms of the scalar and pseudoscalar respectively. The normalization factor $\mathcal{N}^{\prime}$ is chosen such that $\mathcal{Z}[0]=1$. The generating functional is therefore

$$
\begin{aligned}
& \mathcal{Z}\left[\eta, \bar{\eta}, j_{\sigma}, j_{\pi_{a}}\right]= \\
& \mathcal{N}^{\prime} e^{i \int d^{4} x N\left(-\frac{s^{2}}{2 g}\right)} \int \mathcal{D} \sigma \mathcal{D} \pi e^{\int d^{4} x N^{0}\left[-\frac{1}{2 g} \sigma^{2}-\frac{1}{2 g} \pi_{a}^{2}\right]} e^{i \int d^{4} x \sqrt{N}\left[\frac{s}{g} \sigma\right]} \mathcal{Z}_{F}[\eta, \bar{\eta}] e^{i \int d^{4} x\left(j_{\sigma} \sigma+j_{\pi_{a}} \pi^{a}\right)}
\end{aligned}
$$

with $\mathcal{D} \pi=\Pi_{a} \mathcal{D} \pi_{a}$ and

$$
\mathcal{Z}_{F}[\eta, \bar{\eta}]=\int \mathcal{D} \bar{\Psi} \mathcal{D} \Psi e^{i \int d^{4} x[\bar{\Psi} K \Psi+\bar{\eta} \Psi-\bar{\Psi} \eta]}
$$

concerns the functional integration over the quark fields with

$$
K=i \not \beta-m+\frac{1}{\sqrt{N}}\left(\sigma+i \pi \gamma_{5}\right)
$$

With the usual shift

$$
\begin{aligned}
& \Psi^{\prime}=\Psi+K^{-1} \eta \\
& \bar{\Psi}^{\prime}=\bar{\Psi}+\bar{\eta} K^{-1}
\end{aligned}
$$

which leaves the integration measure invariant one obtains after integrating over the fermionic fields

$$
\mathcal{Z}\left[\eta, \bar{\eta}, j_{\sigma}, j_{\pi_{a}}\right]=e^{i \int d^{4} x \bar{\eta} K^{-1} \eta} e^{N T r \ln K} e^{i \int d^{4} x\left(j_{\sigma} \sigma+j_{\pi_{a}} \pi^{a}\right)}
$$

where

$$
\operatorname{Tr} \ln K=\operatorname{Tr} \ln (i \not \beta-m)+i \sum_{n=1}^{\infty} \frac{1}{n} N^{\frac{-n}{2}} \operatorname{Tr}\left[(i \not \partial-m)^{-1}\left(\sigma+i \pi \gamma_{5}\right)\right]^{n}
$$


The full generating functional is

$$
\mathcal{Z}\left[\eta, \bar{\eta}, j_{\sigma}, j_{\pi_{a}}\right]=\mathcal{N} \int \mathcal{D} \sigma \mathcal{D} \pi e^{i \int d^{4} x \bar{\eta} K^{-1} \eta+j_{\sigma} \sigma+j_{\pi_{a}} \pi^{a}} e^{S_{e f f}}
$$

where the effective action

$$
S_{e f f}=\sqrt{N} S_{1}+\frac{1}{2} N^{0} S_{2}+\frac{1}{3 \sqrt{N}} S_{3}+\frac{1}{4 N} S_{4}+\ldots
$$

shows explicitly how the the $N$-counting scheme enters in the different contributions in terms of $n$-point functions, $S_{n}$, see Fig. 1. One has

$$
\begin{aligned}
& S_{1}=\int d^{4} x \frac{\sigma s}{g}-i \operatorname{Tr}[Y] \\
& S_{2}=-\frac{1}{g} \int d^{4} x\left[\sigma^{2}+\pi_{a}^{2}\right]+i \operatorname{Tr}[Y]^{2} \\
& S_{3}=-i \operatorname{Tr}[Y]^{3} \\
& S_{4}=i \operatorname{Tr}[Y]^{4}
\end{aligned}
$$

with the definition $Y=\left[(i / \partial-m)^{-1}\left(\sigma+i \pi \gamma_{5}\right)\right]$. To one quark loop level, $n$ indicates the number of external mesonic fields, $S_{n}$ corresponds to tree level contributions in terms of mesonic fields. In leading order of $1 / N$, one obtains the gap equation by calculating the stationary point of the effective action at $\langle\sigma\rangle=0$. The term $S_{2}$ yields the two point functions, $S_{3}$ and $S_{4}$ the three and four point functions in leading order $1 / N$. To obtain the next-to-leading order corrections in a $1 / N$ expansion of the gap equation and quark and meson propagators it is enough to consider terms up to $S_{4}$. For decay processes involving three external mesonic lines, for instance, one would need to calculate $S_{5}$ as well to describe the next-to-leading order corrections in $1 / N$.

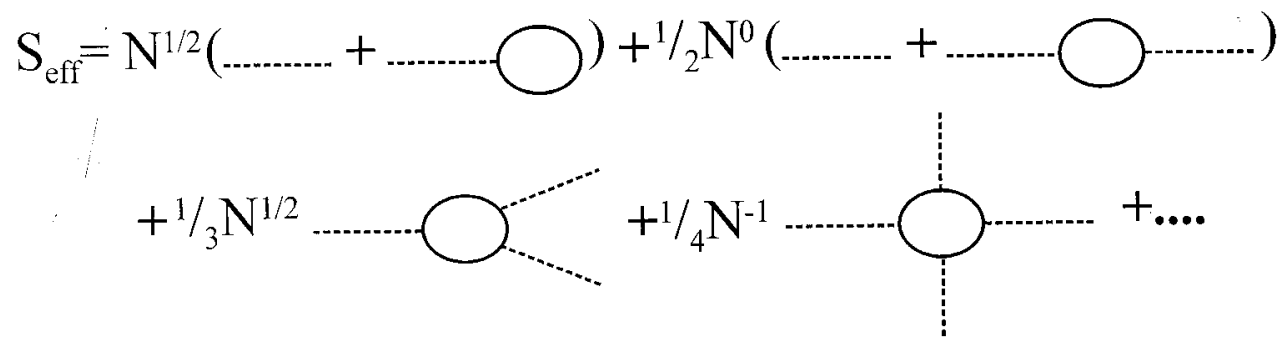

Figure 1. The effective action with the explicit dependence on $\mathrm{N}$ of the several one-loop quark contributions. The dashed lines represent the meson fields. To the $N^{1 / 2}$ term contributes only the scalar meson; the propagator term, of order $N^{0}$, stands for the pion and the sigma mesons; the three point function proportional to $N^{1 / 2}$ involves either three scalars or one scalar coupled to two pions; the four point function of order $N^{-1}$ stands for either all pions or all scalars or two pions and two scalars.

Let us now proceed to calculate the next-to-leading order in $1 / N$ corrections to the gap equation, quark and meson propagators. In eqs. 18-24 we write down the formal expressions and later proceed with their explicit 
calculation. For that one takes the unperturbed Lagrangian to be the one containing up to quadratic mesonic fields, and respective sources $j_{\sigma}$ and $j_{a \pi}$ and treat perturbatively the higher field powers, according to a perturbative expansion in $1 / N$, as

$$
\mathcal{Z}\left[\eta, \bar{\eta}, j_{\sigma}, j_{\pi_{a}}\right]=\mathcal{N} \int \mathcal{D} \sigma \mathcal{D} \pi e^{-i \int d^{4} x\left[\bar{\eta} K^{-1} \eta\right]} e^{i S_{2 \sigma} \sigma} e^{i S_{2 \pi}} e^{i S_{i n t}} e^{i \int d^{4} x\left[j_{\sigma} \sigma+j_{\pi_{a}} \pi^{a}\right]}
$$

where

$$
\begin{gathered}
S_{i n t}=\frac{1}{3 \sqrt{N}} S_{3}+\frac{1}{4 N} S_{4}+\ldots \\
e^{i S_{i n t}}=1+\frac{i}{3 \sqrt{N}} S_{3}+\frac{i}{4 N} S_{4}-\frac{1}{18 N} S_{3} S_{3}+\mathcal{O}\left(1 / N^{2}\right) .
\end{gathered}
$$

The one point function in next to leading order in $1 / N$ is obtained by deriving once with respect to the scalar meson source (the contribution from the pseudoscalar source vanishes) and requiring that the expectation value of the scalar field vanishes in the new vacuum,

$$
\begin{aligned}
& \left.\frac{\delta}{\delta j_{\sigma}\left(x_{1}\right)} \mathcal{Z}\left[\eta, \bar{\eta}, j_{\sigma}, j_{\pi_{a}}\right]\right|_{j=0}= \\
& i \mathcal{N} \int \mathcal{D} \sigma \mathcal{D} \pi e^{-i \int d^{4} x\left[\bar{\eta} K^{-1} \eta\right]} e^{i S_{2}^{\sigma}} e^{i S_{2}^{\pi}} \\
& {\left.\left[1+\frac{i}{3 \sqrt{N}} S_{3}+\ldots\right] \sigma\left(x_{1}\right) e^{i \int d^{4} x\left[j_{\sigma} \sigma+j_{\pi_{a}} \pi^{a}\right]}\right|_{j=0}} \\
& =i \mathcal{N} \int \mathcal{D} \sigma \mathcal{D} \pi e^{i S_{2 \sigma} e^{i S_{2 \pi}}\left[1+\frac{i}{3 \sqrt{N}} S_{3}+\ldots\right] \sigma\left(x_{1}\right)=0}
\end{aligned}
$$

where the generic $j$ stays for all sources. After performing all allowed contractions of the fields one obtains contributions up to the next to leading order in $N$ to the one-point function, see also eq. 25 below. The subleading contribution is depicted in Fig. 2. At this stage it is interesting to compare the selfconsistency condition imposed by the gap equation on the vacuum expextation value of the scalar field with the quark mass obtained from the quark propagator at next to leading order in $1 / N$. In leading order the constituent quark mass coincides with the vacuum expectation value of the scalar field. In order to obtain the quark propagator one takes functional derivatives of $\mathcal{Z}\left[\eta, \bar{\eta}, j_{\sigma}, j_{\pi_{a}}\right]$ with respect to the fermionic sources $\eta$ and $\bar{\eta}$,

$$
\begin{aligned}
& \left.\frac{\delta^{2}}{\delta \eta\left(x_{1}\right) \delta \bar{\eta}\left(x_{2}\right)} \mathcal{Z}\left[\eta, \bar{\eta}, j_{\sigma}, j_{\pi_{a}}\right]\right|_{j=0}= \\
& -\mathcal{N} \int \mathcal{D} \sigma \mathcal{D} \pi \frac{\delta^{2}}{\delta \eta\left(x_{1}\right) \delta \bar{\eta}\left(x_{2}\right)}\left[e^{-i \int d^{4} x\left[\bar{\eta} K^{-1} \eta\right]} e^{i S_{2}^{\sigma}} e^{i S_{2}^{\pi}} e^{i S_{i n t}}\right] \\
= & -\mathcal{N} \int \mathcal{D} \sigma \mathcal{D} \pi\left[-i K^{-1}\left(x_{1}-x_{2}\right)\right] e^{i S_{2}^{\sigma}} e^{i S_{2}^{\pi}} e^{i S_{i n t}}
\end{aligned}
$$


By expanding $\left[-i K^{-1}\left(x_{1}-x_{2}\right)\right]=(1+Y)^{-1}(i \not \partial-m)^{-1}$, in powers of $Y$ up to order $Y^{2}$, and expanding the exponential in $S_{\text {int }}$ up to linear terms in $S_{\text {int }}$, one obtains

$$
\begin{aligned}
& \left.\frac{\delta^{2}}{\delta \eta\left(x_{1}\right) \delta \bar{\eta}\left(x_{2}\right)} \mathcal{Z}\left[\eta, \bar{\eta}, j_{\sigma}, j_{\pi_{a}}\right]\right|_{j=0}= \\
& -i \mathcal{N} \int \mathcal{D} \sigma \mathcal{D} \pi e^{i S_{2}^{\sigma}} e^{i S_{2}^{\pi}}\left[1+\frac{1}{\sqrt{N}} Y+\frac{1}{N} Y^{2}\right](i \not \partial-m)^{-1} e^{i S_{i n t}}
\end{aligned}
$$

where the first term in square brackets is the leading order inverse quark propagator, the term linear in $Y$ multiplied by $S_{\text {int }}$ (the $S_{3}$ part of it) yields the Hartree contribution and the term in $Y^{2}$ the Fock contribution to the next to leading order inverse quark propagator, see Fig. 3. The Fock terms renormalize the quark wave function and the Hartree terms contribute to the constant mass value in the gap equation, compare tadpole diagram of Fig. 3 with Fig. 2. Therefore the quark mass is precisely given by the gap equation when the fermion propagator has a pole, that is when eq. 21 is satisfied.

$$
\mathrm{G}^{(1)}\left(\mathrm{x}_{1}\right)=\mathrm{N}^{-1 / 2} \cdots \cdots
$$

Figure 2. The next to leading order in $N$ to the gap equation. The internal meson line stands for a pion or a sigma.

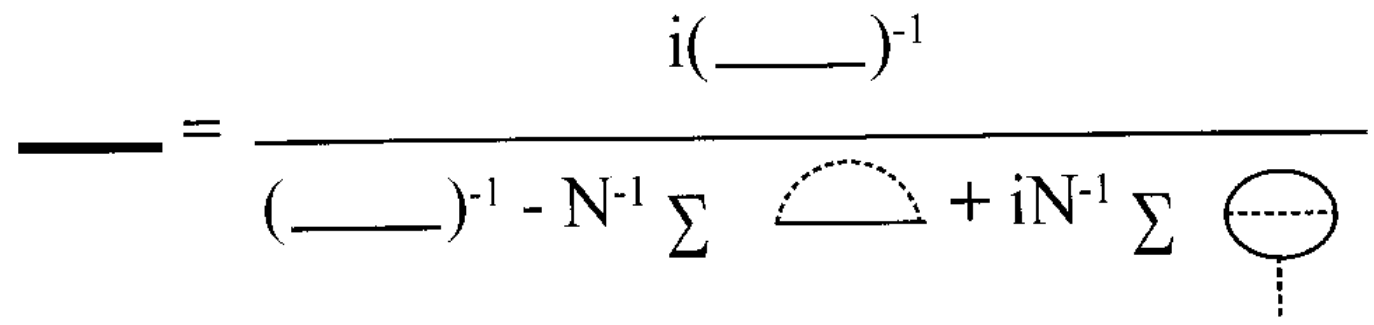

Figure 3. The quark propagator to the next to leading order in the $1 / N$ expansion. The thin lines denote fermion propagators in the dominant order, the dashed internal lines stand for pion and scalar insertions. The external line of the tadpole is a scalar.

The meson propagators are then obtained by deriving twice with respect to the mesonic sources. For instance the scalar propagator is

$$
\begin{aligned}
& \left.\frac{\delta^{2}}{\delta j_{\Phi_{i}}\left(x_{1}\right) \delta j_{\Phi_{j}}\left(x_{2}\right)} \mathcal{Z}\left[\eta, \bar{\eta}, j_{\sigma}, j_{\pi_{a}}\right)\right|_{j=0}= \\
& -\mathcal{N} \int \mathcal{D} \sigma \mathcal{D} \pi e^{-i \int d^{4} x\left[\bar{\eta} K^{-1} \eta\right]} e^{i S_{2}^{\sigma}} e^{i S_{2}^{\pi}} \\
& {\left.\left[1+\frac{i}{3 \sqrt{N}} S_{3}+\frac{i}{4 N} S_{4}-\frac{1}{18 N} S_{3} S_{3}\right] \sigma\left(x_{2}\right) \sigma\left(x_{1}\right) e^{i \int d^{4} x\left[j \sigma \sigma+j_{i \pi} \pi^{i}\right]}\right|_{j=0}} \\
& =-\mathcal{N} \int \mathcal{D} \sigma \mathcal{D} \pi e^{i S_{2 \sigma} \sigma} e^{i S_{2 \pi}}\left[1+\frac{i}{3 \sqrt{N}} S_{3}+\frac{i}{4 N} S_{4}-\frac{1}{18 N} S_{3} S_{3}\right] \Phi_{i}\left(x_{2}\right) \Phi_{j}\left(x_{1}\right)
\end{aligned}
$$


where $\Phi_{i}(x)$ stands for $\sigma(x)$. The pion propagator is obtained with $\Phi_{i}(x)=\pi_{i}(x)$, the corresponding Feynman diagrams are shown in Fig. 4. For the explicit evaluation of the diagrams we use Pauli-Villars regularization with two covariant regulators, one for the quark loop momentum, $\Lambda$, and one for the meson loop momentum. All needed integrals, $I_{i}, i=1, \ldots 4$, are given in the Appendix. We do not need to solve explicitly the meson loop integrals, since we are only interested in showing that the symmetry relations are preserved.

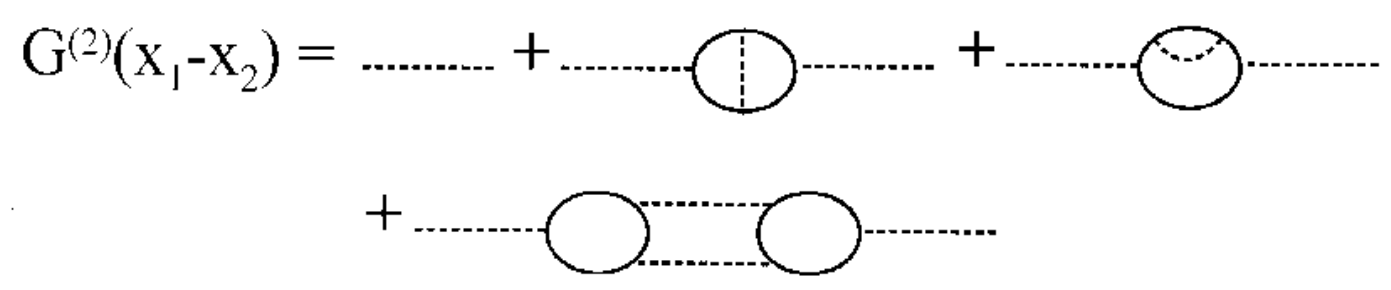

Figure 4. The meson propagator up to next to leading order in the $1 / N$ expansion. In the present work we consider only the pion propagator. The internal line stands in the upper two bubbles for a pion and a scalar and in the lower graph for a pion and a scalar insertion. The same types of diagrams emerge for the calculation of the weak pion decay constant, if one interprets the simple dashed line as well as one of the external lines in the bubble diagrams to be the axial vector current and the other the pseudoscalar meson.

Explicit evaluation of expression (21) yields for the gap equation

$$
\left.\frac{\delta}{\delta j_{\sigma}\left(x_{1}\right)} \mathcal{Z}\left[\eta, \dddot{\pi},\left.\right|_{\sigma},\left.\right|_{\pi_{-}}\right]\right|_{\mid=\prime}=\frac{\mathbb{1}-\mathbb{1}}{\mathcal{G}}-\mathcal{G},(\mathbb{1})-\mathcal{G}_{\pi}(\mathbb{1})-\mathcal{G}_{\sigma}(\mathbb{1})=\prime
$$

with $\Sigma_{0}(m)=G G_{0}(m)=8 m g I_{1}$ the quark self energy of leading order and $\Sigma_{1}(m)=G\left(G_{\pi}(m)+G_{\sigma}(m)\right)$ the quark loops with mesonic insertions, of next to leading order in $1 / N$. The equation

$$
\Sigma(m)=m=\hat{m}+\Sigma_{0}(m)+\Sigma_{1}(m)
$$

must be solved selfconsistently for the same value of $m$, in all terms, as already pointed out in [10]. One has

$$
G_{\pi}(m)=-2 \delta_{i j} \int \frac{d^{4} k}{(2 \pi)^{4}} \int \frac{d^{4} p}{(2 \pi)^{4}} \stackrel{\circ}{S}_{\pi}(p) \operatorname{Tr}_{D}\left[S(k) i \gamma_{5} S(k-p) i \gamma_{5} S(k)\right]
$$

with the quark propagator

$$
S(k)=\frac{i}{\not k-m}
$$

and the inverse pion propagator in leading order

$$
\stackrel{\circ}{S}_{\pi}^{-1}(p)=\frac{\hat{m}}{G m}+4 p^{2} I_{2}(p)
$$

leading to 


$$
G_{\pi}(m)=-24 m \int \frac{d^{4} p}{(2 \pi)^{4}} \stackrel{\circ}{S}_{\pi}(p)\left[-I_{2}(0)+p^{2} I_{3}(0, p)\right]
$$

after summation over the isospin indices. This is the diagram of Fig. 2 with the internal meson line being a pion.

For the quark loop with one scalar meson insertion one obtains

$$
G_{\sigma}(m)=2 \int \frac{d^{4} k}{(2 \pi)^{4}} \int \frac{d^{4} p}{(2 \pi)^{4}} \stackrel{\circ}{S}_{\sigma}(p) \operatorname{Tr}_{D}[S(k) S(k-p) S(k)]
$$

where the inverse scalar propagator in leading order is

$$
\stackrel{\circ}{S}_{\sigma}^{-1}(p)=\frac{\hat{m}}{G m}+4\left(p^{2}-4 m^{2}\right) I_{2}(p)
$$

One obtains

$$
G_{\sigma}(m)=8 m \int \frac{d^{4} p}{(2 \pi)^{4}} \stackrel{\circ}{S}_{\sigma}(p)\left[I_{2}(0)+2 I_{2}(p)+\left(4 m^{2}-p^{2}\right) I_{3}(0, p)\right]
$$

Now we turn to the calculation of the pion propagator, using eq.24. We present the corrections to the selfenergy of the pion $\Pi^{i j}(q)=\sum_{l} P_{l}^{i j}(q)$ with

$$
P_{0}^{i j}(q)=\delta_{i j}\left(24 I_{1}-12 q^{2} I_{2}\left(q^{2}\right)\right)
$$

being the lowest order contribution. The sum of all next to leading order terms $\Pi_{i j}^{1}(q)$ contains the following terms

$$
P_{1}^{i j}(0)=4 \delta^{i j} \int \frac{d^{4} p}{(2 \pi)^{4}} \stackrel{\circ}{S}_{\pi}(p)\left\{2 I_{2}(p-q)-q^{2} p^{2} I_{4}(p, q, p+q)\right\}
$$

which is the first bubble diagram in Fig. 4, with internal pion line;

$$
\begin{aligned}
& P_{2}^{i j}(q)=-24 \delta^{i j} \int \frac{d^{4} p}{(2 \pi)^{4}} \stackrel{\circ}{S}_{\pi}(p) \\
& \left\{I_{2}(p-q)+I_{2}(0)-2 p \cdot q I_{3}(p, q)-q^{2} I_{3}(0, q)+q^{2} p^{2} I_{4}(0, p, q)\right\}+\delta^{i j} \frac{G_{\pi}}{m}
\end{aligned}
$$

which is the second bubble diagram in Fig. 4, with internal pion line;

$$
P_{3}^{i j}(q)=2 \delta^{i j} \int \frac{d^{4} p}{(2 \pi)^{4}} \stackrel{\circ}{S}_{\sigma}(p)\left\{4 I_{2}(p-q)+2 q^{2}\left(4 m^{2}-p^{2}\right) I_{4}(q, p, p+q)\right\}
$$


which is the first bubble diagram in Fig. 4, with internal ;scalar meson line

$$
\begin{aligned}
& P_{4}^{i j}(q)=-8 \delta^{i j} \int \frac{d^{4} p}{(2 \pi)^{4}} \stackrel{\circ}{S}_{\sigma}(p)\left\{I_{2}(p-q)-2 I_{2}(0)-2 p \cdot q I_{3}(p, q)-q^{2} I_{3}(0, q)\right. \\
& \left.-\left(4 m^{2}-p^{2}\right) I_{3}(0, p)-q^{2}\left(4 m^{2}-p^{2}\right) I_{4}(0, p, q)\right\}+\delta^{i j} \frac{G_{\sigma}}{m}
\end{aligned}
$$

which is the second bubble diagram in Fig. 4, with internal scalar meson line;

$$
P_{5}^{i j}=-16 \delta^{i j} \int \frac{d^{4} p}{(2 \pi)^{4}} \stackrel{\circ}{S}_{\sigma}(p-q) \stackrel{\circ}{S}_{\pi}(p)\left\{16 m^{2}\left[I_{2}(p-q)-p \cdot q I_{3}(p, q)\right]^{2}\right\}
$$

which is the last diagram in Fig. 4, where the two internal meson lines are a pion and a scalar, respectively.

To demonstrate the appearence of the Goldstone mode, we compare the quark selfenergy eq.26 to the pion selfenergy $\Pi^{i j}(q)$ and use the identity

$$
\stackrel{\circ}{S}_{\sigma}(p) \stackrel{\circ}{S}_{\pi}(p)=\frac{\stackrel{\circ}{S}_{\sigma}(p)-\stackrel{\circ}{S}_{\pi}(p)}{16 m^{2} I_{2}\left(p^{2}\right)} .
$$

In the chiral limit, $\hat{m} \rightarrow 0$ one gets at $q^{2}=0$

$$
\Pi^{1}(0)=\frac{\Sigma^{1}}{m G}
$$

where $\Pi^{1}(0)$ are all the contributions to the selfenergy of the pion except the leading one, and we use the notation $\Pi^{i j}(q)=\delta_{i j} \Pi(q)$ Therefore the whole relation, including terms up to next to leadind order in $N$ reads

$$
\Sigma=m G \Pi(0)
$$

The inverse pionic Green's function

$$
S_{\pi}^{-1}(q)=G^{-1}-\Pi(q)
$$

has therefore a zero at $q^{2}=0$ when the new gap equation $\Sigma=m$ is fulfilled, verifying the Goldstone theorem. See also discussion after eq. 60 below.

To calculate the weak decay constant $f_{\pi}$ one incorporates in the model an axial current, connected to an external source. In this case the operator $K$ will contain this current

$$
K=i \not \partial-m+\frac{1}{\sqrt{N}}\left(\sigma+i \pi \gamma^{5}+\sqrt{N} \gamma^{\mu} \gamma^{5} \tau^{a} j_{\mu 5 a}\right)
$$

and the new term appears in all terms of the effective action. Only terms linear in $j_{\mu 5 a}$ contribute to the corrections up to leading order in $N$ of $f_{\pi}$. Diagrammatically one obtains the contributions shown in fig.4. We show next that 
the Ward Identity relating the axialvector - pseudoscalar bubble $A(q)$ to the pseudoscalar - pseudoscalar bubble $\Pi(q)$,

$$
A(q)=A_{\mu} q^{\mu}=(m-\hat{m}) / G-m \Pi(q)
$$

with the notation $A^{i j}=\delta_{i j} A(q)$ which holds at leading order in $1 / N$, holds at next to leading order too, which ensures that symmetry relations are preserved. The right hand side of this equation is just the inverse of the pion propagator multiplied by $m$. At next to leading order of $1 / N$ there are several contributions to the bubbles $A(q)$ and $\Pi(q)$, as shown in Fig. 4.

$$
A_{l}(q)=A_{l \mu}(q) q^{\mu}, l=0, \ldots 5
$$

with $A_{0}(q)$ the leading order contribution

$$
A_{0}(q)=G_{0}(m)-4 m N\left[2 I_{1}-q^{2} I_{2}(q)\right]
$$

and the remaining terms follow the same order as for $P_{l}(q)$ in eqs. 35-39. The diagrams $A_{1}(q)$ and $A_{2}(q)$ correspond to the two distinct possibilities of having a pion propagator insertion in the quark loop, $A_{3}(q)$ and $A_{4}(q)$ are the equivalent diagrams for scalar propagator insertions and $A_{5}(q)$ denotes the diagram which couples the external fields to two off-shell mesonic propagators, a pion and a scalar. For diagram $A_{1}(q)$ we obtain

$$
\begin{aligned}
A_{1}^{i j}(q)= & -\sum_{l, l^{\prime}} \operatorname{Tr} \tau_{i} \tau_{l} \tau_{j} \tau_{l^{\prime}} \delta_{l l^{\prime}} i N \int \frac{d^{4} p}{(2 \pi)^{4}} \int \frac{d^{4} k}{(2 \pi)^{4}} \\
& \left\{\stackrel{\circ}{S}_{\pi}(p) \operatorname{Tr}_{D} i \gamma_{5} S(k-q) \frac{i \not h \gamma_{5}}{2} S(k) i \gamma_{5} S(k-p) i \gamma_{5} S(k-p-q)\right\}
\end{aligned}
$$

In order to derive the Ward identity (eq.44) we make use of the equality

$$
\not h=-(\not k-\not h-m)+(\not k+m)-2 m
$$

in all $A_{l}(q)$ diagrams. We have then

$$
A_{1}(q)=-2 R^{\pi}(q)-m P_{1}(q)
$$

where 


$$
\begin{aligned}
R_{i j}^{\pi}(q)= & -4 m N \delta_{i j} i \int \frac{d^{4} p}{(2 \pi)^{4}} \stackrel{\circ}{S}_{\pi}(p)\left[I_{2}(p-q)-p \cdot q I_{3}(p, q)\right] \\
A_{2}^{i j}(q)= & -2 N \sum_{l, l^{\prime}} T r \tau_{i} \tau_{l} \tau_{l^{\prime}} \tau_{j} \delta_{l, l^{\prime}} i \int \frac{d^{4} k}{(2 \pi)^{4}} \int \frac{d^{4} p}{(2 \pi)^{4}} \stackrel{\circ}{S}_{\pi}(p) \\
& \left\{\operatorname{Tr}_{D} i \gamma_{5} S(k) i S(k-q) \frac{i \not q \gamma_{5}}{2} \gamma_{5} S(k) i \gamma_{5} S(k-p)\right\} \\
= & \left(G_{\pi}(q)+6 R^{\pi}(q)-m P_{2}(q)\right) \delta_{i j}
\end{aligned}
$$

$$
\begin{aligned}
A_{3}^{i j}(q)= & -2 N \delta_{i j} i \int \frac{d^{4} k}{(2 \pi)^{4}} \int \frac{d^{4} p}{(2 \pi)^{4}} \stackrel{\circ}{S} \sigma(p) \\
& \operatorname{Tr}_{D}\left\{S(k-q) i \not q \gamma_{5} S(k) S(k-p) i \gamma_{5} S(k-p-q)\right\} \\
= & \left(2 R^{\sigma}(q)-m P_{3}(q)\right) \delta_{i j}
\end{aligned}
$$

$$
R_{i j}^{\sigma}(q)=4 N m \delta_{i j} \int \frac{d^{4} p}{(2 \pi)^{4}} \stackrel{\circ}{S}_{\sigma}(p)\left[I_{2}(p)+\left(p \cdot q-q^{2}\right) I_{3}(p, q)\right]
$$

$$
\begin{aligned}
A_{4}^{i j}(q)= & -2 N \operatorname{Tr} \tau_{i} \tau_{j} i \int \frac{d^{4} k}{(2 \pi)^{4}} i \int \frac{d^{4} p}{(2 \pi)^{4}} \stackrel{\circ}{S}_{\sigma}(p) \\
& \operatorname{Tr}_{D}\left\{S(p) i \gamma_{5} S(k-p) \frac{i \not h}{2} \gamma_{5} S(k) S(k-p)\right\} \\
= & \left(G_{\sigma}(q)+2 R^{\sigma}(q)-m P_{4}(q)\right) \delta_{i j}
\end{aligned}
$$

$$
A_{5}^{i j}(q)=i \int \frac{d^{4} p}{(2 \pi)^{4}} \stackrel{\circ}{S}_{\pi}(p) \stackrel{\circ}{S} \sigma_{\sigma}(p-q) T_{1}^{i l}(p, q) T_{2}^{j l}(p, q)
$$

where $T_{1}^{i l}(p, q),\left(T_{2}\right)^{j l}(p, q)$ are the two quark triangles, coupling the axialvector, (pseudoscalar) to the two off-shell mesonic lines, respectively

$$
T_{1}^{i l}(p, q)=2 N \delta_{i l} i \int \frac{d^{4} k}{(2 \pi)^{4}} \operatorname{Tr}_{D} S(k-q) i \not h \gamma_{5} S(k) i \gamma_{5} S(k-p)
$$




$$
T_{2}^{j l}(p, q)=2 N \delta_{j l} i \int \frac{d^{4} k}{(2 \pi)^{4}} \operatorname{Tr}_{D} i \gamma_{5} S(k) i \gamma_{5} S(k-q) S(k-p)
$$

The triangle $T_{1}^{i l}(p, q)$ splits into the difference of the two leading order inverse propagators $\stackrel{\circ}{S}_{\pi}^{-1}(p)-\stackrel{\circ}{S}_{\sigma}^{-1}(p-q)$ and one gets

$$
A_{5}(q)=-4\left(R^{\pi}(q)+R^{\sigma}(q)\right)-m P_{5}(q) .
$$

Summing up all contributions leads to

$$
\begin{aligned}
A & =q^{\mu} \sum_{i} A_{i}=G_{0}(m)+G_{\pi}(m)+G_{\sigma}(m)-m \sum_{i} P_{i} \\
& =(m-\hat{m}) / G-m \sum_{i} P_{i}
\end{aligned}
$$

using the result for the gap equation, eq.25. This is the desired Ward identity.

The pion weak decay constant $f_{\pi}$ is related to $A(q)$ by

$$
A(q)=q^{2} f_{\pi} / g_{\pi}=m S_{\pi}^{-1}(q)
$$

where $S_{\pi}^{-1}(q)$ is the inverse pion propagator in next to leading order, see eq. 43 . The quantity $g_{\pi}$ is the pion quark - antiquark coupling.

It is now a simple matter to show that the Goldberger-Treiman relation $g_{\pi}(0)=m / f_{\pi}(0)$ is fulfilled to next to leading order in $1 / N$ in the chiral limit. For this one has to make a low momentum expansion of $A(q)$ up to $q^{2}$ order to isolate the Goldstone pole in the pion propagator $S_{\pi}(0)$. The residue at the pole is identified with $g_{\pi}^{2}(0)$.

Note that in $P_{0}(q)$ (leading order) and in $P_{2}(q), P_{4}(q)$ appear $G_{0}(m), G_{\pi}(m)$ and $G_{\sigma}(m)$, which cancel in the evaluation of $A(q)$, see eq. 59. The remaining terms in $A(q)$ are

$$
\begin{aligned}
& A(q)=-4 m i \int \frac{d^{4} p}{(2 \pi)^{4}} \stackrel{\circ}{S}_{\pi}(p)\left\{-4 I_{2}(p-q)+12 p \cdot q I_{3}(p, q)+6 q^{2} I_{3}(0, q)\right. \\
& \left.-q^{2} p^{2}\left(I_{4}(p, q, p+q)-6 I_{4}(0, p, q)\right)\right\} \\
& -4 m i \int \frac{d^{4} p}{(2 \pi)^{4}} \stackrel{\circ}{S}_{\sigma}(p)\left\{4 I_{2}(p)+4 p \cdot q I_{3}(p, q)+2 q^{2} I_{3}(0, q)\right. \\
& +q^{2}\left(4 m^{2}-p^{2}\right)\left(I_{4}(q, p, p+q)+2 I_{4}(0, p, q)\right\} \\
& +16 m i \int \frac{d^{4} p}{(2 \pi)^{4}} \stackrel{\circ}{S}_{\sigma}(p-q) \stackrel{\circ}{S_{\pi}}(p)\left\{16 m^{2}\left[I_{2}(p-q)-p \cdot q I_{3}(p, q)\right]^{2}\right\} .
\end{aligned}
$$


The $I_{4}$ integrals come with $q^{2}$ factors, so it is sufficient to keep only the leading order terms of the $I_{4}$ expansions. The expansions of the $I_{3}$ and $I_{2}$ integrals will generate constant (in $q$ ), $p \cdot q$ and $q^{2}$ terms in the integrand of $A(q)$ The constant terms add up to zero, after using relation (40) in the leading order contribution of the last integral of (61). The terms linear in $p \cdot q$ will vanish since the $p$-integration is odd. Terms quadratic in $p \cdot q$, like for instance from the square term of the last integral lead to $q^{2}$ terms after $p$-integration, due to Lorentz invariance and because the $I_{i}$ integrals are non-singular at $q^{2}=0$. Therefore the low momentum expansion of $A(q)$ starts with a term proportional to $q^{2}$ and one obtains finally the desired relation $g_{\pi}=m / f_{\pi}$ in the chiral limit.

During the development of the present work we became aware of ref. [10] which addresses the same problem with basically the same conclusions. We however hope that the essential differences, as the explicit form of the $1 / N$ expansion given here, and the explicit use of Ward identities, may be a useful tools for further research.

\section{Appendix. The main loop integrals}

List of the main quark-loop integrals, with $\Delta(k)=\frac{1}{k^{2}-m^{2}}$

$$
\begin{gathered}
I_{1}=i \int_{\Lambda} \frac{d^{4} k}{(2 \pi)^{4}} \Delta(k), \\
I_{2}\left(q_{1}\right)=i \int_{\Lambda} \frac{d^{4} k}{(2 \pi)^{4}} \Delta(k) \Delta\left(k+q_{1}\right), \\
I_{3}\left(q_{1}, q_{2}\right)=i \int_{\Lambda} \frac{d^{4} k}{(2 \pi)^{4}} \Delta(k) \Delta\left(k+q_{1}\right) \Delta\left(k+q_{2}\right), \\
I_{4}\left(q_{1}, q_{2}, q_{3}\right)=i \int_{\Lambda} \frac{d^{4} k}{(2 \pi)^{4}} \Delta(k) \Delta\left(k+q_{1}\right) \Delta\left(k+q_{2}\right) \Delta\left(k+q_{3}\right) .
\end{gathered}
$$

\section{References}

[1] Y. Nambu, G. Jona-Lasinio: Phys. Rev. 122, 345 (1961).

[2] F. Gross, J. Milana: Phys. Rev. D 43, 2401 (1991).

[3] W. Marciano, H. Pagels: Phys. Rep. 36, 137 (1978).

[4] U. Vogel, W. Weise: Progr. Part. Nucl. Phys. 7, 199 (1991).
[5] T. Hatsuda and T. Kunihiro: Phys. Rep. 247, 221 (1994);

J. Bijnens: Phys. Rep. 265369 (1996);

V. Bernard, A.H. Blin, B. Hiller, Y.P. Ivano, A.A. Osipov, U.-G. Meißner: Annals of Phys. (NY) bf 249, 499 (1996).

[6] N. -W. Cao, C. M. Skakin, W. -D. Sun: Phys. Rev. C C 46, 2535 (1992).

[7] P.P. Domitrovich, D. Buchers, H. Muther: Phys. Rev. C 48, 413 (1993). 
[8] E. Quack, S. P. Klevansky: Phys. Rev. C 49, 3283 (1994);

P. Zhuang, J. Hüfner, S. P. Klevansky: Nucl. Phys. A 576, 525 (1994);

P. Zhuang, J. Hüfner, S. P. Klevansky, H. Von: Annals of Phys. 234, (225 1994).
[9] V. Dmitrasinovic, H-J. Schulze, R. Tegen, R. H. Lemmer: Ann. of Phys. 238, 332 (1995).

[10] E. N. Nikolov, W. Broniowski, C. V. Christov, G. Ripka, K. Goeke: Nucl. Phys. A 608, 411 (1996). 